\title{
Preliminary Cosmic Ray Results from the HAWC's Eye Telescopes
}

\author{
F. Rehbein, ${ }^{a, *}$ R. Alfaro, ${ }^{b}$ J. Audehm, ${ }^{a}$ T. Bretz, ${ }^{a}$ O. Chaparro-Amaro, ${ }^{c}$ G. Do, ${ }^{a}$ \\ F. J. González, ${ }^{d}$ M. M. González, ${ }^{d}$ A. Iriarte, ${ }^{d}$ J. Martínez-Castro, ${ }^{c}$ \\ M. Martínez-Felipe, ${ }^{c}$ F. Maslowski, ${ }^{a}$ Y. F. Pérez-Araujo, ${ }^{d}$ M. Schaufel, ${ }^{a}$ \\ J. Serna-Franco, ${ }^{b}$ F. Tischbein ${ }^{a}$ and I. Torres $^{e}$ \\ a Physics Institute 3, RWTH Aachen University, \\ Sommerfeldstraße 16, 52074 Aachen, Germany. \\ ${ }^{b}$ Instituto de Física, Universidad Nacional Autónoma de México, \\ Ciudad de México, Mexico. \\ ${ }^{c}$ Centro de Investigación en Computación, Instituto Politécnico Nacional, \\ Ciudad de México, Mexico. \\ ${ }^{d}$ Instituto de Astronomía, Universidad Nacional Autónoma de México, \\ Ciudad de México, Mexico. \\ ${ }^{e}$ Instituto Nacional de Astrofísica, Óptica y Electrónica, \\ Puebla, Mexico. \\ E-mail: florian.rehbein@rwth-aachen.de
}

The compact imaging air-Cherenkov telescope HAWC's Eye was developed to operate with the High-Altitude Water Cherenkov Gamma-Ray Observatory (HAWC). The combination of both detection techniques in a hybrid configuration provides a significant improvement in energy and angular resolution, aiming for improved measurements of the cosmic ray composition above $10 \mathrm{TeV}$ and contributing to the physics program of the observatory. Preliminary results of the first hybrid measurements of the cosmic ray spectrum are presented. After the first HAWC's Eye telescope was brought to the HAWC site in 2017, a second telescope was successfully commissioned in 2019. Two measurement nights have since then recorded the data used in this analysis. The HAWC's Eye events were successfully synchronized with the events recorded from the extensive air-shower array HAWC and further used to characterize the hybrid system. A complete simulation of the hybrid configuration was used to develop algorithms to reconstruct the energy and arrival direction of proton-induced air showers. Those algorithms were successfully applied to the measured cosmic ray events to verify the performance of the hybrid detection. The spectrum reconstructed with HAWC's Eye is consistent with the spectrum reconstructed solely from the coincident HAWC data.

$3^{\text {th }}$ International Cosmic Ray Conference (ICRC 2021)

July 12th-23rd, 2021

Online - Berlin, Germany

\footnotetext{
*Presenter
} 


\section{Introduction}

During the last decades, numerous experiments studied the composition and energy spectrum of the constant flux of ionized particles from space, called cosmic rays. Since the flux of these cosmic rays is steeply falling with energy, large ground-based detectors are needed to measure the highest energies of up to $10^{20} \mathrm{eV}$. Such ground-based detectors can not detect cosmic rays directly due to their interactions with the Earth's atmosphere. The result of such interactions are large cascades of secondary particles, known as extensive air showers.

Ground-based particle arrays directly detect the secondary particles forming the shower front. These arrays are composed of numerous stations that cover a large area. Combining data from multiple stations enables the reconstruction of the shower axis, while the number of secondary particles correlates with the particle energy. When traversing atmospheric matter, the charged shower particles emit Cherenkov photons. Imaging air-Cherenkov telescopes (IACTs) detect those photons and thereby can take images of the showers. Since Cherenkov photons are emitted at every stage of the shower, IACTs provide measurements of the complete shower development through the atmosphere.

Combining both detection techniques in a hybrid setup [1] improves the quality of the shower reconstruction, as they provide complementary information of the same shower. Ground-based particle arrays enable a precise reconstruction of the shower axis and position on ground, but only see the showers at one distinct point of their development. On the contrary, IACTs collect Cherenkov photons during the whole shower development, but are left with an ambiguous reconstruction of the shower position on ground: Small-energy showers close to the telescope produce similar images as high-energy showers far away. The hybrid setup helps to solve these ambiguities and thereby improves the reconstruction of the showers.

This paper presents a preliminary reconstruction of the cosmic ray spectrum with the hybrid setup of HAWC and two HAWC's Eye telescopes.

\section{The HAWC's Eye Telescope as an Extension of the HAWC Gamma-Ray Observatory}

The HAWC's Eye telescope [2] is a compact and light-weight IACT based on refractive optics. Since 2019 [3], two HAWC's Eye telescopes serve as an extension of the High Altitude Water Cherenkov Observatory (HAWC). The telescopes feature an enclosed design, utilizing a Fresnel lens with a diameter of $549.7 \mathrm{~mm}$ and a focal length of $502.1 \mathrm{~mm}$. Their pixelized camera is composed of 61 Silicon Photomultipliers (SiPMs) of type SensL MicroFJ-60035-TSV as photosensors, each equipped with a solid light guide to increase their photosensitive area.

The High Altitude Water Cherenkov Gamma-Ray Observatory HAWC is an extensive air shower array located at an altitude of $4100 \mathrm{~m}$ close to the Sierra Negra volcano in Mexico. With a large field of view of almost $2 \mathrm{sr}$ and a high duty cycle of $95 \%$, it is designed to detect gamma-rays in a broad energy range between $100 \mathrm{GeV}$ and $100 \mathrm{TeV}$ [4]. 


\section{Measurement}

The data presented in this paper was recorded in the night of December 19th 2019 with the two HAWC's Eye telescopes located in the center of the HAWC observatory at a mutual distance of $40 \mathrm{~m}$ both pointing to zenith. The weather conditions were ideal due to a moonless and clear night. About 2.5 hours of observation data was taken and successfully synchronized with the HAWC detector by comparing the absolute trigger timestamps of HAWC's Eye with HAWC. With a time window of $100 \mu \mathrm{s}$, close to $90 \%$ of all HAWC's Eye events were successfully assigned to a HAWC trigger flag. This fraction corresponds to a total number of about 90000 synchronized events per telescope. The excess events can be assigned to night sky background and can be removed with a cut on the minimum number of triggered HAWC tanks.

\section{Energy Reconstruction using Hybrid Data}

For this study, 362000 proton-induced air showers were simulated using the program CORSIKA. The key parameters of the CORSIKA configuration are summarized in Table 1. The maximum simulated zenith angle of $8^{\circ}$ is chosen according to the HAWC's Eye field-of-view of about $6.8^{\circ}$. Since the maximum distance of the telescope to the shower core is limited by the size of the Cherenkov light cone on ground, the simulated area is set to $500 \times 500 \mathrm{~m}^{2}$.

Table 1: CORSIKA configuration for the air shower simulations.

\begin{tabular}{lc}
\hline Parameter & Value/Range \\
\hline Energy spectrum slope & -1.5 \\
Energy range & $1 \mathrm{TeV}-100 \mathrm{TeV}$ \\
Zenith angle & $0^{\circ}$ \\
View Cone & $8^{\circ}$ \\
Shower core scattering area & $500 \times 500 \mathrm{~m}^{2}$ \\
Observation height & $4100 \mathrm{~m}$ \\
Cherenkov wavelength range & $250 \mathrm{~nm}-700 \mathrm{~nm}$ \\
Atmospheric model & US standard atmosphere (7) \\
Magnetic field & $27.717 \mu \mathrm{T}$ (hor.); 29.902 $\mu \mathrm{T}$ (vert.) \\
Hadronic interaction model & GHEISHA (low-energy); QGSJET (high-energy) \\
\hline
\end{tabular}

The HAWC's Eye detector simulation is part of the ROOT-based Major Analysis and Reconstruction Software MARS [5]. From the shower images, camera pixels that contain no shower signal are removed with an image cleaning algorithm taking the signal amplitude and arrival time into account. The background-subtracted signal distributions are then parameterized by a set of image parameters, which, for example, includes the total signal size, the center-of-gavity and the standard deviation along the major and minor axis (width and length).

The HAWC array is simulated using the GEANT4-based [6] software package HAWCsim. For all triggered showers, the energy, arrival direction, and shower core position are reconstructed with the standard HAWC algorithms [8, 9]. 
The HAWC parameters are then combined with the HAWC's Eye image parameters for an improved reconstruction. For the energy reconstruction of the triggered hybrid events, the software package ranger [7] is used. Ranger implements the random forest algorithm, a machine learning algorithm based on bootstrapped decision trees. As input, a selection of HAWC's Eye and HAWC parameters has been optimized for the best possible performance.

The correlation of the reconstructed and Monte Carlo energy is shown in Figure 1. The red line indicates a perfect correlation between the two energies. The distribution shows, that the reconstruction overestimates the primary energy at the smallest energies below about $10 \mathrm{TeV}$ due to the lower analysis threshold. At the highest energies above $50 \mathrm{TeV}$, the reconstruction underestimates the true energy due to the limited simulated energy range.

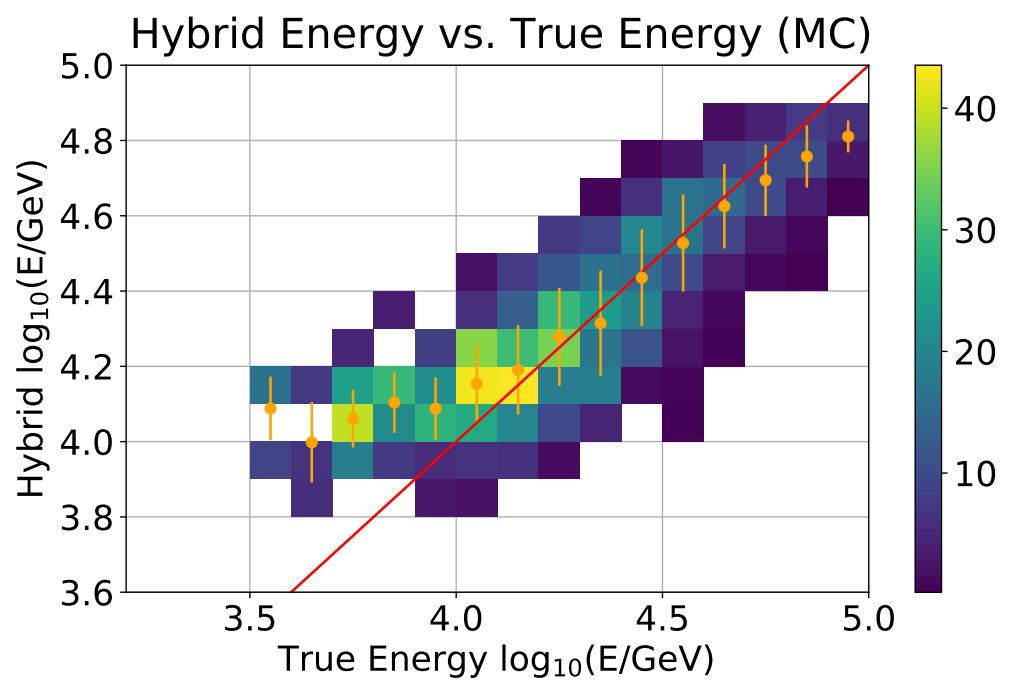

Figure 1: Event distibrution of the reconstructed energy as a function of the Monte Carlo energy for simulated primary protons. The mean and standard deviation for each bin are shown in orange. The red line shows the line of perfect correlation. The energy threshold induces an overestimation at the smallest energies. At the highest energies, the limited simulated energy range causes a negative bias.

The resulting energy resolution, defined as the standard deviation of $\log E_{R F}-\log E_{M C}$ around zero, is shown in Figure 2 as a function of the Monte Carlo energy. As a reference, the energy reconstruction parameter protonlheEnergy obtained from the default HAWC reconstruction, is shown for the same hybrid events in green. As expected, both algorithms show a degraded resolution below the hybrid energy threshold. For all energies below about $10^{4.8} \mathrm{GeV}$, the combination of both detectors provides a clear improvement of the HAWC energy reconstruction. The increase of the resolution above the HAWC curve at the highest energies originates from the discussed bias of the random forest at the edge of the simulated energy range.

It should be noted, that the HAWC standard reconstruction is slightly biased towards smaller energies when applied to the proton-only dataset, since the algorithm is designed to reconstruct a typical cosmic ray composition bias-free. The random forest does not see such a bias, since it was 


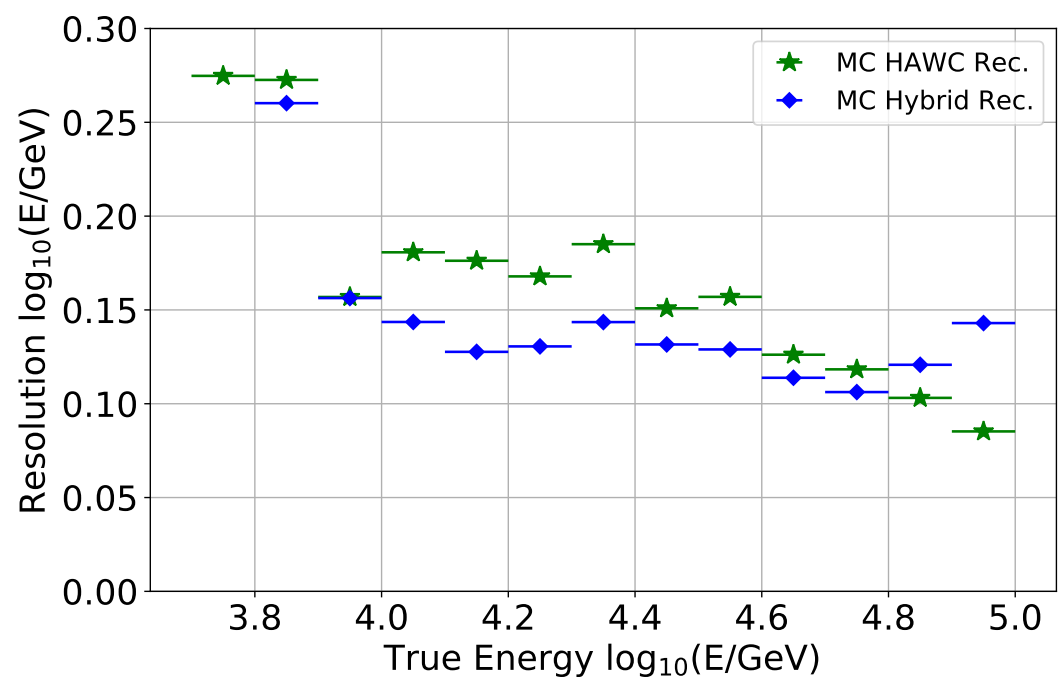

Figure 2: Energy resolution as a function of the Monte Carlo Energy. The green curve represents the energy resolution according to the HAWC default reconstruction for synchronized events, the blue curve shows the hybrid reconstruction of the same events, as discussed in the text. The resolution below $10 \mathrm{TeV}$ and above $50 \mathrm{TeV}$ are biased by the trigger threshold and a limited simulation range respectively.

trained and tested only with protons. Therefore, the application of the trained random forest to real data slightly underestimates the cosmic ray energy (see Section 5).

To correct the measured flux for detector efficiencies, an effective aperture is obtained from the simulation. It is defined as

$$
\mathcal{A}_{\text {eff }}=A_{\text {sim }} \cdot \Omega_{\text {sim }} \cdot \frac{\mathrm{d} N_{\text {trig }}}{\mathrm{d} N_{\text {sim }}}
$$

with the simulated area $A_{\text {sim }}=500 \times 500 \mathrm{~m}^{2}$, the simulated solid angle of $\Omega_{\text {sim }}=0.06 \mathrm{sr}$ corresponding to the maximum zenith angle of $8^{\circ}$, the number of triggered events $\mathrm{d} N_{\text {trig }}$, and the number of simulated events $\mathrm{d} N_{\text {sim }}$ in the respective energy bin.

The effective aperture as a function of the proton energy is depicted in Figure 3.

\section{Cosmic Ray Spectrum}

Using the results of the simulated effective aperture for the hybrid reconstruction, the measured event rates can be transformed into a particle flux. As the spectral index of the simulated spectrum influences the effective aperture and energy migration, correct results can only be obtained simulating the real cosmic ray spectrum. As this spectrum is unknown a priori, the real spectrum is approximated with an iterative procedure. The spectral index of the real spectrum is estimated fitting a power law. Then, the simulated spectrum is re-weighted according to the estimated spectral index and the calculation of the measured cosmic ray spectrum is repeated. After a few iteration steps, the spectral index converges and starts to fluctuate around a certain value. For the final result, 20 iteration steps have been applied to the data. 


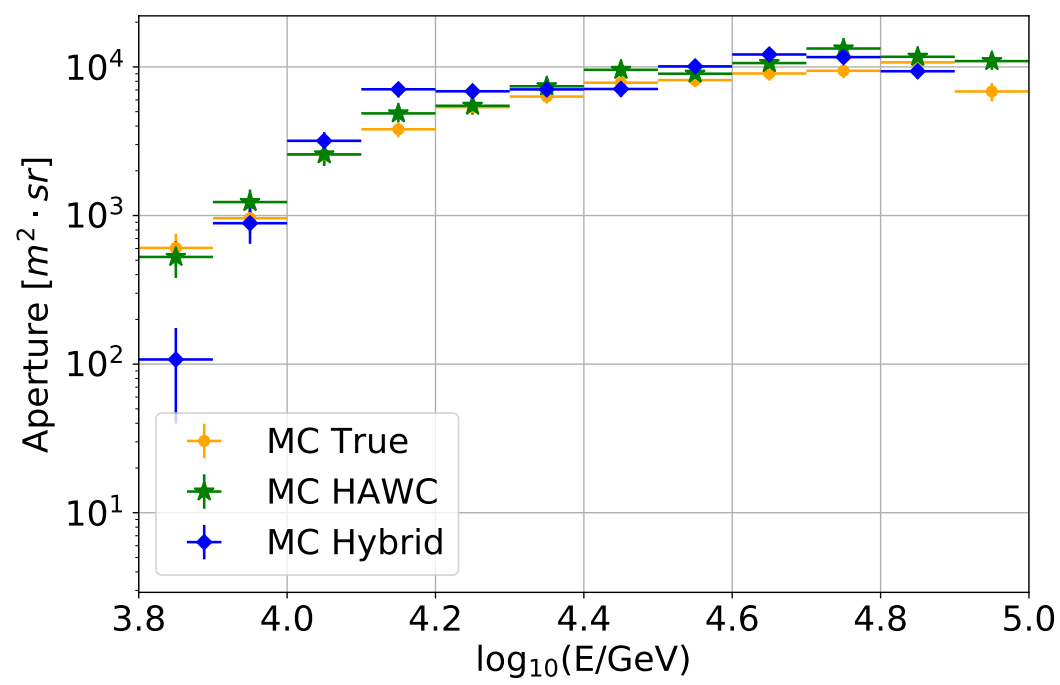

Figure 3: Effective aperture for the detection of proton-induced air showers plottted against different energies: The energy reconstruction of the random forest (blue), the Monte Carlo energy (yellow), and the HAWC energy (green). All curves display the same events. The error bars depict the bin width (horizontal) and the statistical errors (vertical) in each energy bin.

As discussed in Section 4, the random forest used for the hybrid reconstruction was only trained on protons. When applying this algorithm to the measured data, its different mass composition induces a bias in the energy reconstruction. The magnitude of this effect can be estimated by comparing the hybrid reconstructed energies based on protons to the HAWC reconstruction based on a mixed composition. Therefore, the HAWC reconstruction can be used as a reference to correct for the above effect in the hybrid reconstruction. The bias is calculated in the energy region not affected by edge effects. It shows, that the hybrid reconstruction underestimates the logarithmic energy by 0.13 , which will be corrected for in the analysis of the measured data.

The resulting cosmic ray spectrum is compared with a previously published and fully analyzed spectrum of the HAWC observatory in Figure 4. For this, the measured spectrum has been included in the corresponding plot from [10] in which the HAWC result has been compared to data from several other observatories.

The resulting hybrid reconstruction is consistent with the HAWC-only reconstruction of the same hybrid triggered events. However, the reconstructed flux is systematically below the published HAWC spectrum.

As no cuts have been applied to the measured data to select any particle species, all masses contribute to the displayed data. Contrary, the spectra from HAWC and the other observatories are obtained using such selection criteria and only contain proton- and helium-induced showers.

First tests with a small Monte Carlo sample of helium-induced showers show a bias in the hybrid energy reconstruction in the order of 0.05 in logarithmic energy, which is too small to explain the mismatch apparent for the hybrid reconstruction. The two curves, which have been reconstructed assuming only protons, lie in between the displayed HAWC $\mathrm{H}+\mathrm{He}$ spectrum and the recently published HAWC proton-only spectrum [11]. As the mismatch is similar for both 
reconstructed spectra, the requirement for hybrid triggers with HAWC and HAWC's Eye likely induces a selection bias towards heavier elements. Together with different energy thresholds for different particle species, as seen in the small helium sample, this alters the effective aperture. This effect can not easily be estimated and requires detailed simulations with a realistic mixed composition.

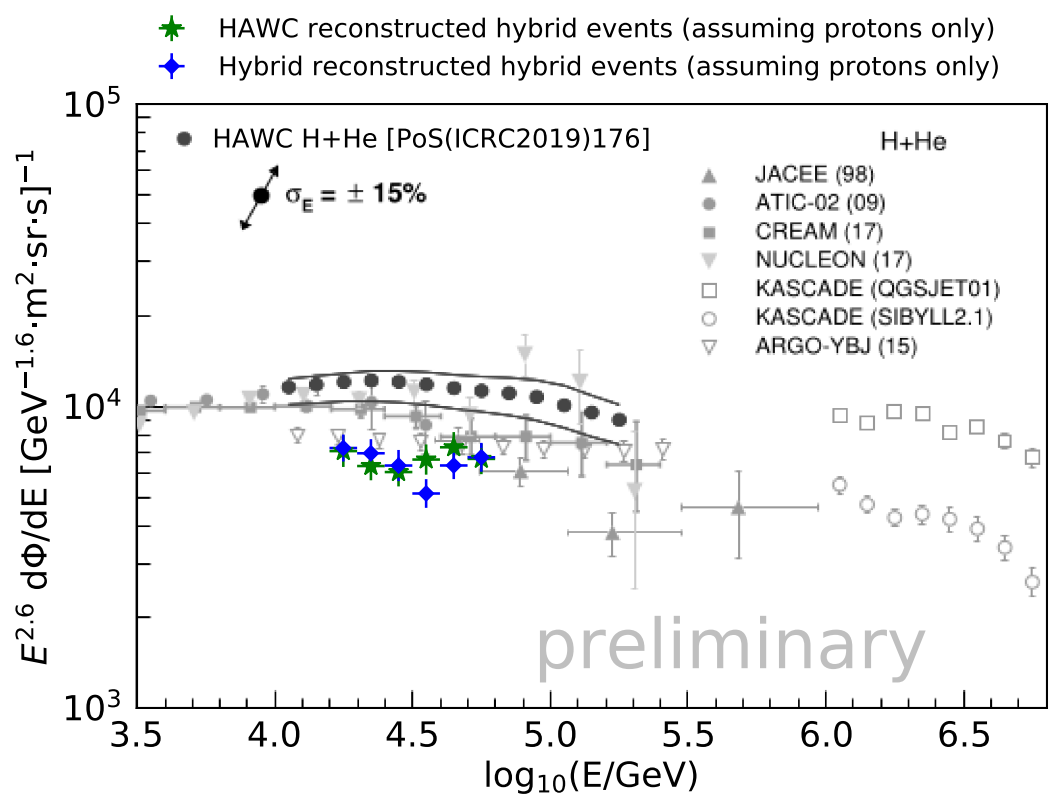

Figure 4: Comparison of the cosmic ray spectrum obtained from a hybrid energy reconstruction with HAWC and HAWC's Eye (green) with previous results from HAWC (black) published in [10]. The blue points show the same triggered hybrid events using a HAWC-only reconstruction. The grey points represent corresponding data from numerous other experiments.

\section{Conclusion}

Two HAWC's Eye telescopes have been used to measure about 2.5 hours of cosmic ray data. About 90000 events per telescope were successfully synchronized with the events measured with the HAWC observatory and used to reconstruct a preliminary cosmic ray spectrum. The obtained spectrum matches well with data that has been published by HAWC and other observatories. However, the resulting spectrum shows a small deviation towards smaller fluxes. The reasons for this shift are not fully understood yet and will be investigated further.

The observed deviations could originate from a systematic overestimation of the light yield, such as an inaccurate simulation of the atmosphere or uncertainties of the camera calibration. More likely, this deviation is an effect of an inaccurate simulation of the mixed composition of cosmic rays.

To investigate the discussed effects, the Monte Carlo dataset should be extended to heavier elements. Possible sources of deviations in the simulation, that could induce a bias in the energy reconstruction, have to be investigated. 


\section{References}

[1] M. Schaufel et al. (HAWC Collaboration), Small size air-Cherenkov telescopes for ground detection arrays - a possible future extension?, In Proceedings of the $35^{\text {th }}$ ICRC, PoS (ICRC 2017) 786.

[2] T. Bretz et al., A compact and light-weighted refractive telescope for the observation of extensive air showers, JINST 13 (2018) P07024.

[3] J. Audehm et al. (HAWC Collaboration), HAWC's Eye - Implementing hybrid detection at the HAWC Gamma-Ray Observatory, In Proceedings of the $36^{\text {th }}$ ICRC, PoS (ICRC 2019) 636.

[4] M. A. Mostafá et al. (HAWC Collaboration), The High Altitude Water Cherenkov Observatory, Nuclear and Particle Physics Proceedings 279-281 (2016) 87-94.

[5] A. Moralejo et al., MARS, the MAGIG Analysis and Reconstruction Software (2009), arXiv:0907.0943.

[6] S. Agostinelli et al., Geant4-a simulation toolkit, Nucl. Inst. Meth. A 506 (2003) 250-303.

[7] M. N. Wright and A. Ziegler, ranger: A Fast Implementation of Random Forests for High Dimensional Data in C++ and R, Journal of Statistical Software 77 (2017) 1.

[8] M. Gussert, A spectral analysis of the Crab Nebula and other sources with HAWC, $\mathrm{PhD}$ thesis, Colorado State University (2016).

[9] V. Joshi, Reconstruction and Analysis of Highest Energy Gamma-Rays and its Application to Pulsar Wind Nebulae, PhD thesis, Ruperto-Carola University of Heidelberg (2019).

[10] J.C. Arteaga-Velázquez and J.D. Álvarez (HAWC Collaboration), The spectrum of the light component of TeV cosmic rays measured with HAWC, In Proceedings of the $36^{\text {th }}$ ICRC, PoS (ICRC 2019) 176.

[11] J.C. Arteaga-Velázquez for the HAWC Collaboration, HAWC measurements of the energy spectra of cosmic ray protons, helium and heavy nuclei in the TeV range, In Proceedings of the $37^{\text {th }}$ ICRC, PoS (ICRC 2021) 374.

\section{Acknowledgments}

We acknowledge the support from DGAPA-UNAM (grants IN111419, IG101320).

The authors thankfully acknowledge the computer resources, technical expertise and support provided by the Laboratorio Nacional de Supercómputo del Sureste de México, CONACYT member of the network of national laboratories.

We acknowledge as well: the funds by the Excellence Initiative of the German federal and state governments and the Helmholtz Alliance for Astroparticle Physics (HAP); the German Academic Exchange Service (DAAD); the FACT collaboration and the Pierre Auger Observatory, as well as the HAWC and IceCube Collaborations; the mechanical and electronic workshops of the RWTH Aachen University for their permanent support. 